Febbraio 2009

$R R-04.09$

Nicola Apollonio, Massimiliano Caramia

A Superclass of Edge-Path-Tree Graphs with Few Cliques

@ by DII-UTOVRM (Dipartimento di Ingegneria dell’Impresa - Università degli Università degli Studi di Roma “Tor Vergata”) This Report has been submitted for publication and will be copyrighted if accepted for publication.

It has been issued as a Research Report for early dissemination of its contents. No part of its text nor any illustration can be reproduced without written permission of the Authors. 


\title{
A Superclass of Edge-Path-Tree Graphs with Few Cliques
}

\author{
Nicola Apollonio* $\quad$ Massimiliano Caramia ${ }^{\dagger}$
}

\begin{abstract}
Edge-Path-Tree graphs are intersection graphs of Edge-Path-Tree matrices that is matrices whose columns are incidence vectors of edge-sets of paths in a given tree. Edge-Path-Tree graphs have polynomially many cliques as proved in [4] and [7]. Therefore, the problem of finding a clique of maximum weight in these graphs is solvable in strongly polynomial time. In this paper we extend this result to a proper superclass of Edge-Path-Tree graphs. Each graph in the class is defined as the intersection graph of a matrix with no submatrix in a set $W$ of seven small forbidden submatrices. By forbidding an eighth small matrix, our result specializes to Edge-Path-Tree graphs.
\end{abstract}

Keywords: Edge-Path-Tree Graphs, Intersection graphs, Maximal Cliques, Graphic Matroids.

\section{Introduction}

In this paper we do not distinguish between a matrix $A \in\{0,1\}^{M \times N}, M$ and $N$ being finite, and the finite family $A=\left(A^{j}\right)_{j \in N}$ of subsets of the finite ground set $M$. This is accomplished by identifying column $A^{j}$ of $A$ with its support in $M$ (and conversely). Recall that the support of a vector $u \in\{0,1\}^{M}$ is the set $\left\{i \in M \mid u_{i}=1\right\}$. Accordingly we use the terms column and member as synonyms and we apply set theoretic operations to the columns of $A$. The intersection graph of $A$ is the graph $L(A)$ with vertex set $N$ in which two vertices $h, j \in N$ are adjacent if $A^{h} \cap A^{j} \neq \emptyset$.

In their paper [4], Golumbic and Jamison introduced and studied Edge-Path-Tree (EPT) graphs defined as intersection graphs of Edge-Path-Tree (EPT) matrices, namely, matrices whose columns are the incidence vectors of edge-sets of paths in a given tree. One of the nicest features of EPT graphs is that they generalize line graphs while retaining the property of possessing polynomially many maximal cliques, i.e., $O\left(n^{2}\right), n$ being the order of the EPT graph, as showed by Monma and Wey in [7]. This fact implies that in an EPT graph a maximum weight clique can be found in strongly polynomial time by running a polynomial time delay algorithm that generates all maximal cliques $[10,6]$.

In this paper we extend the above mentioned results in $[4,7]$ to a proper superclass of EPT graphs: the class of intersection graphs of $\{0,1\}$ matrices whose submatrices are not isomorphic to anyone in

$$
W=\left\{F_{7}, F_{7}^{*}, 3 \mathrm{PC}, 3 \mathrm{PC}_{1}, H_{3,3}, Y_{3,3}, 3 \mathrm{PC}_{2}\right\}
$$

\footnotetext{
*Istituto per le Applicazioni del Calcolo, M. Picone, Via G. Amendola, 122/D 70126 Bari, Italy. nicola.apollonio@uniroma1.it

†Dipartimento di Ingegneria dell'Impresa, Università di Roma "Tor Vergata", Via del Politecnico 1, 00133 Roma, Italy. caramia@disp.uniroma2.it
} 


$$
\begin{aligned}
& \left(\begin{array}{llll}
1 & 1 & 1 & 0 \\
1 & 0 & 1 & 1 \\
1 & 1 & 0 & 1
\end{array}\right) \quad\left(\begin{array}{lll}
1 & 1 & 1 \\
1 & 1 & 0 \\
0 & 1 & 1 \\
1 & 0 & 1
\end{array}\right) \quad\left(\begin{array}{llll}
1 & 1 & 1 & 0 \\
1 & 0 & 0 & 1 \\
0 & 1 & 0 & 1 \\
0 & 0 & 1 & 1
\end{array}\right) \quad\left(\begin{array}{lllll}
1 & 1 & 1 & 0 & 0 \\
1 & 0 & 1 & 1 & 0 \\
1 & 0 & 0 & 1 & 1 \\
1 & 1 & 0 & 0 & 1
\end{array}\right) \quad\left(\begin{array}{lllll}
1 & 1 & 0 & 0 & 1 \\
1 & 1 & 1 & 0 & 0 \\
0 & 1 & 1 & 1 & 0 \\
0 & 0 & 1 & 1 & 1
\end{array}\right) \\
& \begin{array}{lllll}
\mathbf{F}_{7} & \mathbf{F}_{7}^{*} & \mathbf{3 P C} & \mathbf{H}_{3,3} & \mathbf{Y}_{3,3}
\end{array} \\
& \left(\begin{array}{lllll}
1 & 1 & 1 & 0 & 0 \\
1 & 0 & 0 & 0 & 1 \\
0 & 1 & 0 & 1 & 0 \\
0 & 0 & 1 & 0 & 1 \\
0 & 0 & 0 & 1 & 1
\end{array}\right) \\
& \left(\begin{array}{lllll}
1 & 1 & 0 & 0 & 0 \\
1 & 0 & 1 & 0 & 0 \\
1 & 0 & 0 & 1 & 0 \\
0 & 1 & 0 & 0 & 1 \\
0 & 0 & 1 & 0 & 1 \\
0 & 0 & 0 & 1 & 1
\end{array}\right) \\
& \left(\begin{array}{llll}
1 & 1 & 0 & 0 \\
1 & 0 & 1 & 0 \\
1 & 0 & 0 & 1 \\
0 & 1 & 1 & 0 \\
0 & 1 & 0 & 1 \\
0 & 0 & 1 & 1
\end{array}\right)
\end{aligned}
$$

Figure 1: The eight matrices used in the paper.

the matrices in $W$ being defined in Figure 1 (see also the end of Section 2 for a discussion). The extension is proper in the sense that by forbidding one more matrix, namely, the matrix $Q_{6}$ in Figure 1, the result specializes to EPT graphs and leads to a bound of $5 q / 3$ for the number of the maximal cliques in a graph with $q$ edges. Our result relies on two powerful tools introduced in [4], namely, the notions of strong Helly number (a strengthening of the Helly Property) and of 3-pie (an extension of the notion of triangle in a graph), which we now discuss briefly.

Let $A \in\{0,1\}^{M \times N}$ be an EPT matrix with $n:=|N|$ columns underlain by a tree $T$ with $m:=|M|$ edges. The strong Helly number of $A$ is the least $h \in \mathbb{N}$ such that for any $k \geq h$ pairwise intersecting columns of $A$ there are $h$ among them whose intersection equals the intersection of the $k$ chosen columns. Golumbic and Jamison proved that $A$ has strong Helly number three, that is, said explicitly, for each $K \subseteq N,|K| \geq 3$, such that the members of $\left(A^{k}\right)_{k \in K}$ are pairwise intersecting, there are three indices $k_{1}, k_{2}, k_{3} \in K$ such that

$$
\cap_{k \in K} A^{k}=A^{k_{1}} \cap A^{k_{2}} \cap A^{k_{3}} \text {. }
$$

For a maximal clique $K$ in the EPT graph $G=L(A)$, the members of $\left(A^{k}\right)_{k \in K}$ are pairwise intersecting by definition of clique. Thus, either $\cap_{k \in K} A^{k} \neq \emptyset$ and the paths spanned by the $A^{k}$ 's go through the same edge of $T$, or $\cap_{k \in K} A^{k}=A^{k_{1}} \cap A^{k_{2}} \cap A^{k_{3}}=\emptyset$ for some three indices $k_{1}, k_{2}, k_{3} \in K$ and (as proved by Golumbic and Jamison) the corresponding paths all go through the center of some claw (a $K_{1,3}$ ) of $T$ and each one contains exactly two of the three edges of the claw (any such pair of edges is referred to as a slice in [4]). In the former case $K$ is an edge-clique while in the latter one $K$ is a claw-clique. Monma and Wey proved in [7] that the number of edgecliques is $O(n)$. Thus, the number of maximal cliques has the order of the number of claw-cliques of $G$.

In view of (1) a natural upper bound for the latter number is the number of 3-elements subsets of $N$ times the maximum number $t$ of claw-cliques containing a given 3 -element set. It is clear that each claw-clique is uniquely determined by any three paths containing, respectively, one of the slices of the corresponding claw in $T$. Therefore, $t=1$ and the number of claw-cliques is at most $\left(\begin{array}{l}n \\ 3\end{array}\right)=O\left(n^{3}\right)$. Monma and Wey improved this bound to $O\left(n^{2}\right)$.

By a result of [2] stating that a $\{0,1\}$-matrix $A$ has strong Helly number $h \geq 2$ if and only if 
$A$ does not contain the matrix $O_{h+1}=J_{h+1}-I_{h+1}$ as submatrix, $\left(J_{h}\right.$ and $I_{h}$, being the all ones and the identity matrix of order $h$, respectively) it follows straightforwardly that $\{0,1\}$-matrices with no $F_{7}^{*}$ submatrix have strong Helly number three (because for $h \geq 4, F_{7}^{*}$ is a submatrix of $O_{h}$ ). Therefore, we can still speak of edge-clique and claw-cliques as in [4].

However, we need to understand what is meant by a claw-clique in the more general case of $W$-free matrices, i.e., matrices with no submatrix in $W$. This is accomplished in Lemma 1 and Lemma 2. In Theorem 1 and in Theorem 2 we prove that intersection graphs of $W$ - and $W \cup\left\{Q_{6}\right\}$ free matrices have $O\left(n^{3}\right)$ and $O(q)$ maximal cliques, respectively, where $q$ is the number of edges of the intersection graph. Corollary 1 specializes the result to EPT graphs. We stress here the fact that the extension is possible because it is possible to give vertex-free proofs of the results in $[4,7]$, namely, proofs that do not exploit arguments involving vertices of the underlying tree realization of an EPT graph.

Notation and preliminaries All the matrices dealt with in this paper are binary matrices, i.e., $\{0,1\}$-matrices and throughout the rest of the paper rows and columns are indexed by the finite sets $M$ and $N$, respectively, with $m=|M|$ and $n=|N|$. The transpose of a binary matrix $A$ is denoted by $A^{*}$. Accordingly, $A^{*}$ is identified with the family of the supports of the rows of $A$ (such a family is the so called dual family). Two matrices $A$ and $A^{\prime}$ are isomorphic if $A^{\prime}=Q A P$ for some two permutation matrices $Q$ and $P$. For $I \subseteq M$ and $J \subseteq N, A_{I}^{J}$ is the matrix obtained from $A$ by deleting the columns whose indices are not in $J$ and the rows whose indices are not in $I$. If $I=M$ we set $A_{I}^{J}=A^{J}$. Analogously, if $J=N$ we set $A_{I}^{J}=A_{I}$. In particular $A^{\{j\}}$ and $A_{\{i\}}$ are abridged into $A^{j}$ and $A_{i}$, respectively. A submatrix (subfamily) of $A$ is any matrix of the form $A_{I}^{J}$ for some $I \subseteq M$ and $J \subseteq N$. We also put $A(J)=\cap_{j \in J} A^{j}$. Thus, for $I \subseteq M, A^{*}(I)=\cap_{i \in I} A_{i}$. A binary matrix not containing any submatrix isomorphic to one of non-isomorphic matrices in the set $D=\left\{H_{1}, \ldots, H_{r}\right\}$, will be referred to both as an $H_{1}, \ldots, H_{r}$-free and $D$-free matrix. We also say that $A$ has no $H$ submatrix to mean that $A$ has no submatrix isomorphic to $H$. A 3pie in $A$ is a subset $J=\left\{j_{1}, j_{2}, j_{3}\right\} \subseteq N$ such that the three columns of $A^{J}$ pairwise intersect and $A(J)=\emptyset$. Each of $A^{j_{1}} \cap A^{j_{2}}, A^{j_{2}} \cap A^{j_{3}}$ and $A^{j_{1}} \cap A^{j_{3}}$ is called a branch of the 3-pie. We observe explicitly that the branches of a 3-pie are pairwise disjoint. For a 3-pie $J \subseteq N$ in $A$ let $S(J)=\left\{t \in N \mid A^{j} \cap A^{t} \neq \emptyset, j \in J\right\}$. Moreover, let

$$
S_{0}(J)=\left\{t \in S(J) \mid A^{t} \cap\left(\left(A^{j_{1}} \cap A^{j_{2}}\right) \cup\left(A^{j_{2}} \cap A^{j_{3}}\right) \cup\left(A^{j_{1}} \cap A^{j_{3}}\right)\right)=\emptyset\right\}
$$

and

$$
S_{2}(J)=S(J) \backslash S_{0}(J) .
$$

By Golumbic and Jamison's argument if $K$ is a claw-clique of the intersection graph of an EPT matrix $A$, then the set $\left\{k_{1}, k_{2}, k_{3}\right\}$ in (1) is a 3-pie. Furthermore, for each $j \in K \backslash\left\{k_{1}, k_{2}, k_{3}\right\}, A^{j}$ intersects exactly two branches of the pie. This fact is in general no longer true for intersection graphs of $W$-free matrices: take for instance the matrix $Q_{6}$ with columns left to right indexed by $0,1,2,3,4 ; Q_{6}$ is a $W$-free matrix (see the discussion preceding Corollary 1 ); moreover, $J=\{1,2,3\}$ is a 3-pie in $Q_{6}$ and $0 \in S(J)$; however $A^{0} \subseteq A^{1} \Delta A^{2} \Delta A^{3}$, where $\Delta$ denotes symmetric difference. This consideration justifies the introduction of the sets $S_{0}(J)$ and $S_{2}(J)$. Lemma 1 and Lemma 2, up to technicalities, show that the case of the $Q_{6}$ is general for $W$-free matrices, that is, either $A^{j}$ intersects $A^{k_{1}} \Delta A^{k_{2}} \Delta A^{k_{3}}$ or $A^{j}$ behaves as it were a path going to the center of some claw in a tree. 


\section{Results}

Lemma 1 and Lemma 2 below taken together describe the structure of the set $S(J)$ for a 3 -pie $J$ in a $W$-free matrix $A$. Such a structure is exploited to count the number of claw-cliques in Theorem 1 and in Theorem 2. Lemma 3 provides a bound on the number of edges cliques of intersection graphs of the more general $F_{7}^{*}$-free matrices. Throughout the rest of the paper $U(J)$ will denote $\cup_{j \in J} A^{j}$.

Lemma 1 Let $J$ be a 3-pie in a $W$-free matrix $A$. Then $t \in S_{2}(J)$ if and only if $A^{t}$ intersects exactly two branches of $J$. Moreover, the members of $A^{S_{2}(J)}$ are pairwise intersecting and $S_{2}(J)=$ $S_{2}(L)$ for each 3-pie $L$ in $A^{S_{2}(J)}$.

Proof. Possibly after renumbering we may suppose that $J=\{1,2,3\}$. Let $B^{1}=A^{1} \cap A^{2}$, $B^{2}=A^{2} \cap A^{3}$ and $B^{3}=A^{1} \cap A^{3}$ be the branches of $J$. For $t \in S(J)$ let $b(t)$ be the number of branches intersected by $A^{t}$. By the definition of $S_{0}(J)$ and $S_{2}(J)$ if $b(t) \geq 1$ then $t \in S_{2}(J)$. Thus, to prove the first part of the lemma it suffices to show that $b(t)=2$ for each $t \in S_{2}(J)$. For no $t \in S_{2}(J), b(t)=3$ otherwise by picking $i(j) \in A^{t} \cap B^{j}, j \in J$ and letting $I=\{i(1), i(2), i(3)\}$ one has that $A_{I}^{J \cup\{t\}}$ is isomorphic to $F_{7}$ contradicting that $A$ is $W$-free. For no $t \in S_{2}(J), b(t)=1$. For, if $A^{t}$ intersects $B^{1}$, say, then $A^{t}$ must intersect $A^{3}$ because $t \in S(J)$. Since $A^{t} \cap B^{2}=A^{t} \cap B^{3}=\emptyset$ (because $b(t)=1$ for the sake of contradiction) it follows that $A^{t} \cap A^{3} \subseteq A^{3} \backslash\left(A^{1} \cup A^{2}\right)$. Pick $i(1) \in A^{t} \cap B^{1}$ and let $i(2)$ and $i(3)$ be arbitrarily chosen in $B^{2}$ and $B^{3}$, respectively. Let $i \in A^{t} \cap A^{3}$ and let $I=\{i, i(1), i(2), i(3)\}$. Thus $A_{I}^{J \cup\{t\}}$ is isomorphic to $3 \mathrm{PC}$ contradicting that $A$ is $W$-free. We conclude that $b(t)=2$ for each $t \in S_{2}(J)$ and the first part of lemma is thus established. The second part is a straightforward consequence of the following claim.

Claim 1 For $j=1,2,3$ there is $\beta(j) \in B^{j}$ such that for each $t \in S_{2}(J)$ if $A^{t}$ intersects $B^{j}$ then it contains $\beta(j)$. Therefore, for each $t \in S_{2}(J) A^{t}$ contains exactly one of the three slices $\{\beta(1), \beta(2)\},\{\beta(1), \beta(3)\}$ and $\{\beta(2), \beta(3)\}$.

Proof of (1). We prove the claim only for $j=1$ as the other cases follow by symmetry. Suppose that the claim is false. Hence there are $s, t \in S_{2}(J)$ and $\alpha(s), \alpha(t) \in B^{1}$ such that $A^{s} \cap\{\alpha(s), \alpha(t)\}=$ $\alpha(s)$ and $A^{t} \cap\{\alpha(s), \alpha(t)\}=\alpha(t)$. By the first part of the lemma $A^{s}$ and $A^{t}$ both intersect exactly one among $B^{2}$ and $B^{3}$. Let us distinguish two cases:

(a) $A^{s}$ and $A^{t}$ intersect the same branch and, without loss of generality, let such a branch be $B^{2}$; (b) $A^{s}$ and $A^{t}$ intersect different branches and, without loss of generality, let $A^{s}$ intersect $B^{2}$ and $A^{t}$ intersect $B^{3}$.

In case (a), let $\gamma(s) \in A^{s} \cap B^{2}$ and $\gamma(t) \in A^{t} \cap B^{2}$ and notice that $\{\alpha(s), \alpha(t), \gamma(s), \gamma(t)\} \subseteq$ $A^{2}$. Necessarily $\gamma(s) \neq \gamma(t)$, otherwise by letting $I=\{\alpha(s), \alpha(t), \gamma(s)\}$ and $L=\{1,2, s, t\}$ one has that $A_{I}^{L}$ is isomorphic to $F_{7}$ contradicting that $A$ is $W$-free. Thus, $\gamma(s) \neq \gamma(t)$. If we let $I=\{\alpha(s), \alpha(t), \gamma(s), \gamma(t)\}$ and $L=J \cup\{s, t\}$ then $A_{I}^{L}$ is isomorphic to $H_{3,3}$ again contradicting that $A$ is $W$-free. Hence, case (a) leads to a contradiction. In case (b) let $\gamma(s) \in A^{s} \cap B^{2}$, $\gamma(t) \in A^{t} \cap B^{3}$ and $I=\{\alpha(s), \alpha(t), \gamma(s), \gamma(t)\}$. Thus, $A_{I}^{L}$ where $L=J \cup\{s, t\}$, is thus isomorphic to $Y_{3,3}$ contradicting once more that $A$ is $W$-free. Hence, case (b) leads to a contradiction as well. Consequently we must conclude that the claim is true and the lemma is thus completely proved.

Lemma 2 Let $J$ be a 3-pie in a $W$-free matrix $A$. Then $C:=A_{U(J)}^{S_{0}(J)}$ is a (possibly empty) chain, that is the members of $C$ are nested. If in addition $A$ is $Q_{6}$-free then $S_{0}(J)$ is empty. 
Proof. Possibly after renumbering we may suppose that $J=\{1,2,3\}$. Let $\beta(1), \beta(2)$ and $\beta(3)$ be as in the Claim 1 of Lemma 1. We first show that $C$ is laminar, namely each two columns of $C$ are either disjoint or nested. Suppose not. Hence, there are $s, t \in S_{0}(J)$ such that $C^{s} \cap C^{t}$, $C^{s} \backslash C^{t}$ and $C^{t} \backslash C^{s}$ are all nonempty. Since $A^{s}$ and $A^{t}$ intersect all members of the 3-pie and both are subsets of $A^{1} \Delta A^{2} \Delta A^{3}$ it follows that one of the following cases applies:

(a) there are $i \in\left(C^{s} \cap C^{t}\right) \cap A^{j}, \alpha(s) \in\left(C^{s} \backslash C^{t}\right) \cap A^{k}$ and $\alpha(t) \in\left(C^{t} \backslash C^{s}\right) \cap A^{k}$, for $j \neq k$ and $j, k \in J$ (without loss of generality $j=1$ and $k=2$ ). In this case let $I=\{i, \alpha(s), \alpha(t), \beta(1)\}$ and $L=\{1,2, s, t\}$;

(b) there are $i \in\left(C^{s} \cap C^{t}\right) \cap A^{j}, \alpha(s) \in\left(C^{s} \backslash C^{t}\right) \cap A^{k}$ and $\alpha(t) \in\left(C^{t} \backslash C^{s}\right) \cap A^{l},\{j, k, l\}=J$ (without loss of generality $j=1$ and $k=2$ and $l=3)$. In this case let $I=\{i, \alpha(s), \alpha(t), \beta(2), \beta(3)\}$ and $L=J \cup\{s, t\}$.

Since $C^{s} \subseteq A^{s}$ and $C^{t} \subseteq A^{t}$ it follows that in case (a) $A_{I}^{L}$ is isomorphic to a $3 \mathrm{PC}$ while in case (b) it is isomorphic to $3 \mathrm{PC}_{1}$. In either cases we obtain a contradiction that proves that $C$ is laminar. Suppose now that $C$ is nonempty but it is not a chain. Thus $C^{s} \cap C^{t}=\emptyset$ for some $s, t \in S_{2}(J)$. Pick $\alpha(s)$ and $\gamma(s)$ in $C^{s} \cap A^{1}$ and $C^{s} \cap A^{2}$, respectively. Also pick $\alpha(t)$ and $\gamma(t)$ in $C^{t} \cap A^{1}$ and $C^{t} \cap A^{2}$, respectively. Observe that none of them belongs to $A^{3}$. Thus, by setting $I=\{\alpha(s), \alpha(t), \beta(2), \beta(3), \gamma(s), \gamma(t)\}$ and $L=J \cup\{s, t\}, A_{I}^{L}$ is isomorphic to $3 \mathrm{PC}_{2}$ contradicting that $A$ is $W$-free. Finally if $A$ is also $Q_{6}$-free then $S_{0}(J)$ is empty, otherwise, by letting $I=\{\alpha(1), \alpha(2), \alpha(3), \beta(1), \beta(2), \beta(3)\}$, where $\alpha(j) \in C^{s} \cap A^{j}$ and $s \in S_{0}(J)$, one has that $A_{I}^{J \cup\{s\}}$ is isomorphic to $Q_{6}$.

Let $G=L(A)$ for some $W$-free matrix $A$. Following Golumbic and Jamison we say that the maximal clique $K$ of $G$ is an edge-clique if $A(K) \neq \emptyset$; we say that $K$ is claw-clique if $A(K)=\emptyset$. Since $F_{7}^{*}$-free matrices (and hence $W$-free matrices) have strong Helly number 3 , it follows that for each claw-clique $K$ of $G$ there is a 3 -pie $J \subseteq K$ such that $A(K)=A(J)$. We say that $J$ represents $K$.

Lemma 3 Let $G$ be the intersection graph of some $F_{7}^{*}$-free matrix $A$ and let $G$ have $q$ edges. Then the number of edge-cliques of $G$ is at most $n_{1}+q$, where $n_{1}$ is the number of isolated vertices of $G$.

Proof. Let $K$ be an edge-clique of $G=L(A)$. There is some $i \in A(K)$. The matrix $A^{K}$ cannot contain the matrix $O_{3}=J_{3}-I_{3}$ as submatrix (remark that $O_{3}$ is the vertex edge incidence matrix of a triangle). For, if $A_{I}^{J}$ is isomorphic to $O_{3}$ for some $J \subseteq K$ and $I \subseteq M$, then $i \notin I$ and $A_{I \cup\{i\}}^{J}$ is isomorphic to $F_{7}^{*}$. Therefore, $A^{K}$ has strong Helly number 2 by the result in [2]. It follows that if $K$ is not a singleton then $A(K)=A^{s} \cap A^{t}$ for some $s, t \in K$. Moreover, it cannot happen that $A\left(K^{\prime}\right)=A^{s} \cap A^{t}$ for some other edge-clique $K^{\prime}$, otherwise we should conclude $K=K^{\prime}$ contradicting maximality. Therefore, by picking $s(K), t(K) \in K$ such that $A(K)=A^{s(K)} \cap A^{t(K)}$ for each non-singleton edge-clique $K$ one defines an injection from the set of the non-singleton edge-cliques into the set of edges of $G$ and this proves the lemma.

Remark 1 Lemma 3 and its proof imply the following bound on the number of inclusionwise maximal rows of $F_{7}^{*}$-free matrices: an $F_{7}^{*}$-free matrix with $n$ columns has at most $\left(\begin{array}{l}n \\ 2\end{array}\right)$ inclusionwise maximal rows. 
Remark 2 A binary matrix $A$ is Helly if for any collection of pairwise intersecting columns there is some row of $A$ which intersects each of them in a nonzero entry. It is strong Helly if every submatrix of $A$ is strong Helly. Strong Helly matrices were characterized by Ryser (see [5]) and Prisner [8]. Both results assert that $A$ is strong Helly if and only if it is $\mathrm{O}_{3}$-free. After the result in [2], strong Helly matrices are precisely those matrices that have strong Helly number 2. Hence, strong Helly matrices are necessarily $F_{7}^{*}$-free. Prisner proved in [8] that if $A$ is strong Helly then $L(A)$ has more edges than maximal cliques. Thus, Lemma 3 extends the result in [8].

Theorem 1 Let $G$ be the intersection graph of some $W$-free matrix $A$ and let $G$ have $n$ vertices and $q$ edges. Then the number of maximal cliques of $G$ is at most $q+\left(\begin{array}{l}n \\ 3\end{array}\right)$.

Proof. By Lemma 3, it suffices to show that $G$ has at most $\left(\begin{array}{l}n \\ 3\end{array}\right)$ claw-cliques. To accomplish this it suffices to prove that each 3-pie $J$ in $A$ represents at most one claw-clique. Observe that if $J$ represents $K$ then $K \subseteq S(J)$ and $K=\left(K \cap S_{2}(J)\right) \cup\left(K \cap S_{0}(J)\right)$ because $S_{0}(J)$ and $S_{2}(J)$ partition $S(J)$. Suppose, for the sake of contradiction, that $J$ represents $K$ and $K^{\prime}$. By the maximality of $K$ and $K^{\prime}$ we can find $s \in K \backslash K^{\prime}$ and $t \in K^{\prime} \backslash K$ such that $A^{s} \cap A^{t}=\emptyset$. By Lemma 1 , the members of $A^{S_{2}(J)}$ are pairwise intersecting. Thus $s, t \in\left(K \Delta K^{\prime}\right) \cap S_{0}(J)$. It follows that $A^{s} \cap U(J)$ and $A^{t} \cap U(J)$ are both members of $A_{U(J)}^{S_{0}(J)}$ and they are disjoint. Thus $A_{U(J)}^{S_{0}(J)}$ is not a chain contradicting Lemma 2. We conclude that the number of claw-cliques is bounded as stated.

Theorem 2 Let $G$ be the intersection graph of some $W \cup\left\{Q_{6}\right\}$-free matrix $A$. Let $G$ have $q$ edges. Then the number of maximal cliques of $G$ is at most is at most $\frac{5 q}{3}$.

Proof. By Lemma 3 it suffices to prove that the number of claw-cliques of $G$ is at most $\frac{2 q}{3}$. Let us form a collection $\Pi$ by picking for each claw-clique $K$ of $G$ a 3-pie which represents $K$ (if there are several the choice is arbitrary). Let $\pi=|\Pi|$. By the second part of Lemma $2, S_{0}(J)=\emptyset$ for each $J \in \Pi$. Therefore, if $J$ represents $K$ then $K \subseteq S(J)=S_{2}(J)$. As $K$ is maximal and $S_{2}(J)$ is a clique (by Lemma 1), it follows that $K=S_{2}(J)$. Moreover, as each 3-pie $J$ represents at most one claw-clique (by the proof of Theorem 1 ), we conclude that the number of claw-cliques is precisely $\pi$. A set $\{s, t\} \subseteq N$ is called a good pair if it is a subset of some $J \in \Pi$. Let $\beta$ be the number of good pairs and let $\mu$ the maximum number of $J$ 's that contain a good pair. Since there are three good pairs for each $J \in \Pi$ it follows that $3 \pi \leq \mu \beta$. Now observe that if $\{s, t\}$ is a good pair then necessarily $A^{s} \cap A^{t} \neq \emptyset$. Therefore, $G$ contains an edge between $s$ and $t$ for each good pair $\{s, t\}$. Hence, $\beta \leq q$ and $3 \pi \leq \mu q$. Consequently, to complete the proof it suffices to show that $\mu \leq 2$. This is accomplished next. Suppose that $\mu \geq 3$. Thus, there are $j_{1}, j_{2}, j_{3} \in N$ such that $J_{1}=\left\{s, t, j_{1}\right\}, J_{2}=\left\{s, t, j_{2}\right\}$ and $J_{3}=\left\{s, t, j_{3}\right\}$ are in $\Pi$ for some good pair $\{s, t\}$. Possibly after renumbering, $j_{1}=1, j_{2}=2$ and $j_{3}=3$. For $j=1,2,3$, the definition of 3 -pie implies that $A^{j} \cap A^{s} \cap A^{t}=\emptyset$ and hence $A^{j} \cap\left(A^{s} \cup A^{t}\right) \subseteq A^{s} \Delta A^{t}$. Moreover, $A^{1}, A^{2}, A^{3}$ are pairwise disjoint. For, if $A^{1} \cap A^{2} \neq \emptyset$, say, then $2 \in S(J)=S_{2}\left(J_{1}\right)$. Since $A^{2} \cap A^{s} \cap A^{t}=\emptyset$ it follows that $A^{2}$ intersects the two branches $A^{1} \cap A^{s}$ and $A^{1} \cap A^{t}$. Hence, by Lemma $1, J_{1}$ and $J_{2}$ would represent the same claw-clique, contradicting the definition of $\Pi$. We conclude that $A^{1}, A^{2}, A^{3}$ are disjoint subsets of $A^{s} \Delta A^{t}$. Therefore, for $j=1,2,3$, one can pick $\alpha(j) \in A^{j} \cap\left(A^{s} \backslash A^{t}\right)$ and $\gamma(j) \in A^{j} \cap\left(A^{t} \backslash A^{s}\right)$. But then, after setting $I=\{\alpha(1), \alpha(2), \alpha(3), \gamma(1), \gamma(2), \gamma(3)\}$ and $L=\{1,2,3, s, t\}, A_{I}^{L}$ is isomorphic to $3 \mathrm{PC}_{2}$, contradicting that $A$ is $W$-free. 
Relation to EPT graphs To show that Theorem 1 and Theorem 2 actually specialize to EPT graphs we need a few elementary basic notions on binary matroids. Chapters $\mathbf{7}$ and $\mathbf{8}$ of [1] and Chapters $\mathbf{2 0}$ and $\mathbf{2 1}$ of [9] contain all what we need here (the reader is referred also to the textbooks cited therein). With every binary matrix $A$ one can associate the binary matroid $M(A)$ generated by $\left[I_{m}, A\right]$. Such a matroid is defined as the matroid whose circuits are the minimal supports of the vectors in the nullspace of $\left[I_{m}, A\right],\left[I_{m}, A\right]$ being a viewed as a matrix over $G F(2)$. Equivalently, $M(A)$ is the matroid whose circuits are the minimal nonempty members in $\left\{\Delta_{j \in J} A^{j} \cup\{j\} \mid J \in 2^{N}\right\}$. Two binary matrices are $G F(2)$-equivalent if one arises from the other by a sequence of $G F(2)$-pivoting ${ }^{1}$. Any binary matrix $A$ is $G F(2)$-equivalent to itself. $G F(2)$ equivalent matrices generate the same binary matroid and, conversely, if $M(A)=M\left(A^{\prime}\right)$ then $A$ and $A^{\prime}$ are $G F(2)$-equivalent. A minor in $M(A)$ is a matroid of the form $M(C)$ where $C$ is a submatrix of some matrix $A^{\prime}$ which is $G F(2)$-equivalent to $A$. A regular matroid is a binary matroid not containing $M\left(F_{7}\right)$ and $M\left(F_{7}^{*}\right)$ as minors - Tutte's deep characterization of regular matroids asserts that the binary matroid $M$ is regular if and only if each $A$ such that $M=M(A)$ is a regular matrix, namely, it can be turned into a totally unimodular matrix by changing the sign to some of its entries [1,9]. The matrices $3 \mathrm{PC}$ and $3 \mathrm{PC}_{1}$ are not regular [1]. Therefore, if $A$ is regular then $A$ is $\left\{F_{7}, F_{7}^{*}, 3 \mathrm{PC}, 3 \mathrm{PC}_{1}\right\}$-free. A binary matroid is graphic if it is generated by an EPT matrix and it is co-graphic if it generated by the transpose of an EPT matrix [3]. For a graph $G$ and a spanning forest $T$ of $G$ the EPT matrix generated by $T$ is the EPT matrix whose generic column is the edge-sets of the path $C(e, T) \backslash\{e\}$, where for $e \in E(G) \backslash E(T), C(e, T)$ is the unique (fundamental) circuit through $e$ in the graph $(V(G), E(T) \cup\{e\})$. The graphic matroid of $G$ is denoted by $M(G)$ and the co-graphic matroid of $G$ is denoted by $M^{*}(G)$. EPT matrices are regular because they can be signed to become network matrices which are totally unimodular [9] (this amounts to orient the edges of $T$ ). Therefore graphic and co-graphic matroid are regular (since being totally unimodular is preserved under transposition). The matrices $H_{3,3}^{*}$ and $Y_{3,3}^{*}$ are two of the three non isomorphic EPT matrices that generate the graphic matroid of the complete bipartite graph $K_{3,3}$. They are respectively generated by the two non isomorphic spanning tree $H$ and $Y$ of $K_{3,3}$ whose degree sequences are $(1,1,1,1,3,3)$ and $(1,1,1,2,2,3)$, respectively. Therefore, $H_{3,3}$ and $Y_{3,3}$ are $G F(2)$-equivalent matrices. Moreover, it is not hard to see that $3 \mathrm{PC}_{2}$ is $G F(2)$-equivalent to a matrix containing $H_{3,3}$ as submatrix. It follows that if $A$ generates a regular matroid with no $M^{*}\left(K_{3,3}\right)$ minor then $A$ is $W$-free. By another deep result of Tutte graphic matroids are precisely those regular matroids with no $M^{*}\left(K_{3,3}\right)$ and $M^{*}\left(K_{5}\right)$ minors. The matrix $Q_{6}$ is the incidence matrix of the collection formed by the edge sets of the triangles of the $K_{4}$. Hence, $Q_{6}^{*}$ is the EPT matrix of the $K_{5}$ generated by the spanning tree isomorphic to the star $K_{1,4}$. Thus, $M\left(Q_{6}\right)=M^{*}\left(K_{5}\right)$. Therefore, EPT matrices are $W \cup\left\{Q_{6}\right\}$ free matrices. In view of the preceding discussion the following corollary is a straightforward consequence of Theorem 1 and Theorem 2 and the result of Monma and Wey asserting that EPT graphs have at most $2 n$ edge-cliques [7].

Corollary 1 Let $A$ be a binary matrix with $n$ columns which generates a regular matroid with no $M^{*}\left(K_{3,3}\right)$ minor. Then $L(A)$ has $O\left(n^{3}\right)$ maximal cliques. If $A$ is an EPT matrix then $L(A)$

\footnotetext{
${ }^{1}$ Recall that pivoting $A$ over $G F(2)$ on a nonzero entry (the pivot element) means replacing

$$
A=\left(\begin{array}{cc}
1 & a \\
b & D
\end{array}\right) \quad \text { by } \tilde{A}=\left(\begin{array}{cc}
1 & a \\
b & D+b a
\end{array}\right)
$$

where the rows and columns of $A$ have been permutated so that the pivot element is $a_{1,1}$ ([1], p. 69, [9], p. 280).
} 
has at most $\min \{2 n+2 q / 3,5 q / 3\}$ maximal cliques, $q$ being the number of edges of the EPT graph $L(A)$.

As a concluding remark let us justify the use of the symbol $W$ for the set of forbidden submatrices considered in this paper. The bipartite graph $B(A)$ of a matrix $A \in\{0,1\}^{M \times N}$ is the bipartite graph with color classes $M$ and $N$ where $i \in M$ and $j \in M$ are linked if $a_{i, j}=1$. The graphs $B\left(F_{7}\right)$ and $B\left(F_{7}^{*}\right)$ are both isomorphic to a graph called odd wheel with three spokes. The graph $B\left(H_{3,3}\right)$ is isomorphic to an even wheel with four spokes. Thus, by the discussion preceding Corollary 1, for each $H \in W$, either $B(H)$ is a wheel or $H$ is $G F(2)$-equivalent to a matrix $H^{\prime}$ such that $B\left(H^{\prime}\right)$ is either a wheel or contains a wheel as induced subgraph ${ }^{2}$. This justifies the use of prefix $W$.

\section{References}

[1] G. Cornuéjols, Combinatorial Optimization. Packing and Covering. SIAM, CBMS-NSF, Philadelphia (2001).

[2] M. C. Dourado, F. Protti, J. L. Szwarcfiter. On the Strong p-Helly Property. Discrete Applied Mathematics 156 (2008) 1053-1057.

[3] J. -C. Fournier. Hypergraphes de Chaines d'Aretes d'un Arbre. Discrete Mathematics 43 (1983) 29-36.

[4] M. C. Golumbic and R.E. Jamison. The Edge Intersection Graphs of Paths in a Tree. Journal of Combinatorial Theory, Series B 38 (1985) 8-22.

[5] J. Lehel. Helly-Hypergraphs and Abstract Interval Structures. ARS Combinatoria 16 (1983) 239-253.

[6] K. Makino, T. Uno. New algorithms for enumerating all maximal cliques. Proc. 9th Scand. Worksh. Algorithm Theory (SWAT 2004), pp. 260.272. Springer-Verlag, Lecture Notes in Computer Science 3111, 2004.

[7] C. L. Monma and V.K. Wey. Intersection Graphs of Paths in a Tree. Journal of Combinatorial Theory, Series B 41 (1986) 141-181.

[8] E. Prisner. Hereditary clique-Helly graphs. Journal of Combinatorial Mathematics and Combinatorial Computing (1993) 14: 216-220.

[9] A. Schrijver. Theory of Linear and Integer Programming, Wiley, (1986).

[10] S. Tsukiyama, M. ide, H. Ariyoshi and I. Shirakawa. A new algorithm for generating all the maximal independent sets. SIAM Journal on Computing 6 (1977) 505-517.

\footnotetext{
${ }^{2}$ Actually the operation of GF(2)-pivoting $A$ can be carried out directly on $B(A)$, see [1].
} 